\title{
Ab Initio Calculations of X-ray Spectra: Atomic Multiplet and Molecular Orbital Effects in a Multiconfigurational SCF Approach to the L-Edge Spectra of Transition Metal Complexes
}

\author{
Ida Josefsson, ${ }^{\dagger}$ Kristjan Kunnus, ${ }^{\ddagger}$ Simon Schreck, ${ }^{\ddagger}$ Alexander Föhlisch, ${ }^{\ddagger}$ Frank de Groot, ${ }^{\S}$ \\ Philippe Wernet, ${ }^{\ddagger}$ and Michael Odelius ${ }^{* \dagger}$ \\ ${ }^{\dagger}$ Department of Physics, Stockholm University, AlbaNova University Center, 10691 Stockholm, Sweden
${ }^{\ddagger}$ Helmholtz-Zentrum Berlin für Materialien und Energie GmbH, Albert-Einstein-Strasse 15, 12489 Berlin, Germany
${ }^{\S}$ Inorganic Chemistry and Catalysis, Utrecht University, Universiteitsweg 99, 3584 CG Utrecht, Netherlands
}

ABSTRACT: A new ab initio approach to the calculation of X-ray spectra is demonstrated. It combines a high-level quantum chemical description of the chemical interactions and local atomic multiplet effects. We show here calculated L-edge X-ray absorption (XA) and resonant inelastic X-ray scattering spectra for aqueous $\mathrm{Ni}^{2+}$ and $\mathrm{XA}$ spectra for a polypyridyl iron complex. Our quantum chemical calculations on a high level of accuracy in a post-Hartree-Fock framework give excellent agreement with experiment. This opens the door to reliable and detailed information on chemical interactions and the valence electronic structure in $3 \mathrm{~d}$ transition-metal complexes also in transient excited electronic states. As we combine a molecular-orbital description with a proper treatment of local atomic electron correlation effects, our calculations uniquely allow, in particular, identifying the influence of interatomic chemical interactions versus intra-atomic correlations in the L-edge X-ray spectra.

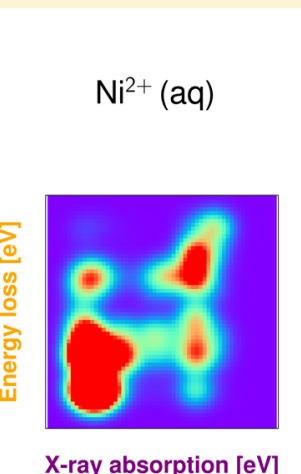

$\mathrm{H}_{2} \mathrm{O}$

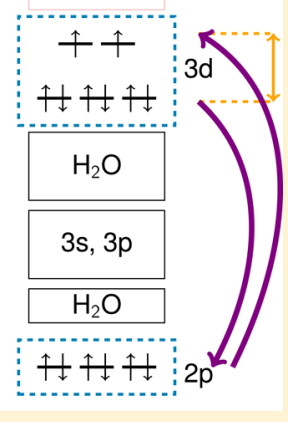

SECTION: Spectroscopy, Photochemistry, and Excited States

I n many energy-related applications, the active chromophores contain transition metal centers, such as the metallo-organic complexes employed in artificial photosynthesis or in dyesensitized solar cells, ${ }^{1}$ for which L-edge resonant inelastic X-ray scattering (RIXS) can be used to map core excitations onto valence excitations. Consequently, we can extract valuable information about the electronic character in specific valenceexcitations. The decay of the core-excited states into valenceexcited states constitute the fluorescence decay channels. Hence, the calculations can also be used to obtain an interpretation of fluorescence yield XA spectra required for a reliable analysis of core-level spectra of transition metal complexes in solution., ${ }^{2,3}$ In future applications, we will be able to tackle realistic models of large metal complexes reaching applications in biochemistry and catalysis.

The field of L-edge X-ray spectrum simulations is undergoing a rapid evolution. Over the last 20 years, semiempirical multiplet calculations have successfully been applied to many molecular as well as solid-state systems. ${ }^{4,5}$ In the case of closedshell ground states, Bethe-Salpeter-based calculations have been used to study systems such as $\mathrm{TiO}_{2} \cdot{ }^{6-8}$ The multichannel multiple scattering code is also able to describe the L-edges of $\mathrm{TiO}_{2}$ and the importance of long-range effects in crystalline systems is shown. ${ }^{9}$ Time-dependent (TD) density functional theory (DFT) calculations have also been applied to $3 \mathrm{~d}^{0}$ transition metal systems, though the combined effect of core-valence electron-electron interactions and spin-orbit couplings seem not yet treated correctly in the results as published so far. ${ }^{10} \mathrm{~A}$ few ab initio calculations have been published for open-shell $3 \mathrm{~d}$ systems: Hidekazu Ikeno and coworkers have developed an ab initio multiplet code based on DFT-CI within the ji-coupling limit. This procedure allows an accurate calculation of $2 \mathrm{p}$ XA spectra of $3 \mathrm{~d}^{0}$ (and $3 \mathrm{~d}^{N}$ ) systems, ${ }^{11}$ starting from a ground state geometry as determined in a DFT calculation. Maurits Haverkort and co-workers perform ab initio cluster calculations, in which they project their initial DFT calculations to localized Wannier orbitals that are subsequently used in a cluster calculation including the full multiplet effects. $^{12}$

L-edge spectroscopy of transition metal compounds is particularly challenging due to their multiconfigurational character and strong spin-orbit coupling. In the restricted active space (RASSCF) method, ${ }^{13}$ a proper treatment of the multiplicity effects, present for transition metals, is guaranteed by the explicit inclusion of static electron correlation through the multiconfigurational wave function. A highly accurate description of the excited states can be obtained when taking dynamic electron correlation into account in a perturbation treatment (CASPT2). ${ }^{14}$ The RASSCF method have great similarities with the methods based on Wannier functions, ${ }^{12}$

Received: September 21, 2012

Accepted: November 19, 2012

Published: November 19, 2012 
and it has a potential advantage in the state-specific orbital optimization in the RASSCF algorithm. In core-excited states, a rehybridization of the molecular orbitals in the presence of the core-hole is automatically included in the treatment.

Our calculations on $\mathrm{Ni}^{2+}\left(\mathrm{H}_{2} \mathrm{O}\right)_{6}$ and $\mathrm{Fe}^{2+}\left(\operatorname{tren}(\mathrm{py})_{3}\right)$ were performed using the MOLCAS-7 software. ${ }^{15}$ In the RASSCF approach, ${ }^{13}$ the multideterminant wave function is built up as excitations from a Hartree-Fock (HF) calculation, which in the case of the core-excitations had a single hole distributed uniformly over the core-orbitals, to generate suitable starting orbitals. The excitations are generated by selecting a set of active spaces of molecular orbitals; RAS1 with a restricted number of holes (the three $2 \mathrm{p}$ orbitals with at most one hole), RAS2 without constraint on occupation (containing the five nominal 3d orbitals), and possibly RAS3 with a restricted number of electrons (e.g., ligand orbitals for MLCT excitation). The remaining orbitals are inactive doubly occupied or empty orbitals. An important aspect for the accuracy of the RASSCF results is that the orbitals in the multideterminant wave function are optimized for the selected valence-excited and core-excited electronic states. Hence, the orbitals we denote $3 \mathrm{~d}$ are molecular orbitals with appropriate hybridization mixing in ligand contributions.

The $\mathrm{Ni}^{2+}\left(\mathrm{H}_{2} \mathrm{O}\right)_{6}$ complex in the high-spin ground state, shown schematically in Scheme 1 , was optimized $(\mathrm{Ni}-\mathrm{O}=$

Scheme 1. Molecular orbital diagram of $\mathrm{Ni}^{2+}\left(\mathrm{H}_{2} \mathrm{O}\right)_{6}{ }^{a}$

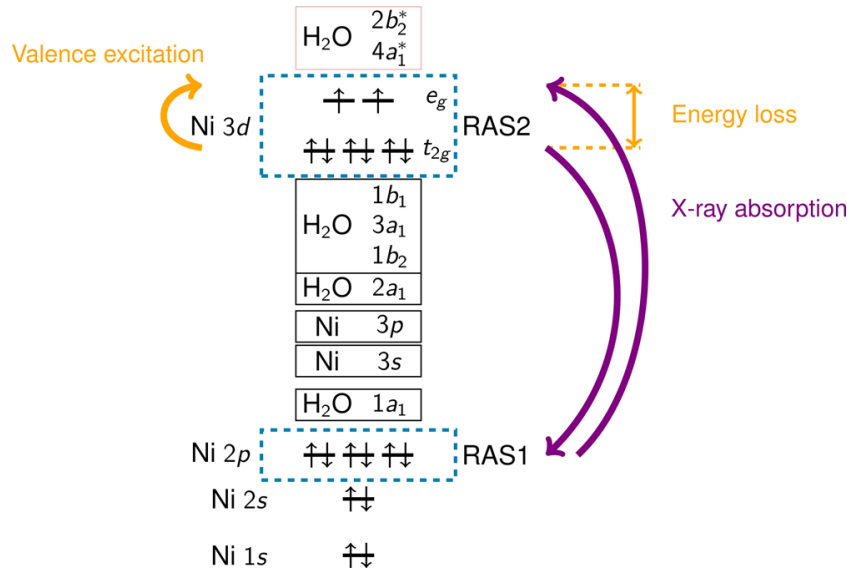

${ }^{a}$ For core excitations ( $\mathrm{Ni} 2 \mathrm{p} \rightarrow \mathrm{Ni} 3 \mathrm{~d}$ ), as well as the subsequent decay into valence-excited states, to be covered, the active space involves $\mathrm{Ni}$ $2 \mathrm{p}$ and $\mathrm{Ni} 3 \mathrm{~d}$, partitioned into two subspaces. RAS1 contains the Ni $2 \mathrm{p}$ orbitals with at most one hole, whereas RAS2 allows all possible electron permutations in the $\mathrm{Ni} 3 \mathrm{~d}$ orbitals. Ligand-to-metal chargetransfer (LMCT) excitations can be accounted for by introducing ligand orbitals in RAS1 or RAS2. By introducing ligand orbitals in a third subspace RAS3 containing at most one electron, we can also model metal-to-ligand charge-transfer (MLCT) excitation.

$2.080 \AA$ ) at the CASPT2 level ${ }^{14}$ with a RAS2 space consisting of the Ni $3 \mathrm{~d}$ orbitals. Scalar relativistic effects were introduced via a spin-free Douglas-Kroll-Hess transformation ${ }^{16,17}$ in conjunction with an all-electron ANO-RCC basis set of VTZP quality. ${ }^{18,19}$ The spin-orbit matrix elements were computed in the state-interaction framework over a basis of RASSCF wave functions. ${ }^{20}$ The vertical transition energies were obtained from state-averaged RASSCF and RASPT2 calculations performed of all resulting singlet and triplet states within each symmetry class in $D_{2 h}$ for the $\mathrm{Ni}^{2+}\left(\mathrm{H}_{2} \mathrm{O}\right)_{6}$ complex. We used a Lorentzian broadening of $0.5 \mathrm{eV}$ at the $\mathrm{L}_{3}$ edge and of $1.0 \mathrm{eV}$ at the $\mathrm{L}_{2}$ edge, motivated by the difference in core-hole lifetimes due to Coster-Kronig (CK) decay, ${ }^{21,22}$ and an overall $0.7 \mathrm{eV}$ Gaussian broadening to account for experimental resolution $(0.4 \mathrm{eV})$ and configurational sampling. To ensure convergence, the core orbitals were constrained to their shape from the underlying HF calculation, and the core excitations were shifted ad-hoc $(-1.85 \mathrm{eV}$ for RASPT2) in comparison to experiment to compensate for limitations in the wave functions. In complementary calculations of the high-intensity transition to the next-lowest triplet core-excited state, the ad-hoc shift was rationalized by (i) avoiding state-averaging (giving a contribution of $0.01 \mathrm{eV}$ ) and (ii) lifting the contraction of the $2 \mathrm{p}$ basis function into a core triple- $\zeta$ basis set (giving an additional contribution of $-0.46 \mathrm{eV}$ ) and (iii) better accounting for the electron correlation by including two ligand orbitals in the RAS1 space (at most one hole) and three ligand orbitals in RAS3 (at most 1 electron), which altogether accounts for $-1.66 \mathrm{eV}$ of the ad-hoc shift.

A Car-Parrinello ab initio molecular dynamics (MD) simulation ${ }^{23,24}$ of $\mathrm{NiCl}_{2}$ solvated in 92 water molecules at ambient conditions was performed using the BLYP functional for the purpose of configurational sampling. The polypyridyl iron $\left[\mathrm{Fe}\left(\operatorname{tren}(\mathrm{py})_{3}\right)\right]^{2+}$ complex was optimized in the singlet and quintet states in DFT (B3LYP) calculations with the TZVP basis set. Both the sampling of Ni L-edge XA spectra and Fe Ledge XA spectra were performed without symmetry using the TZVP basis set and effective core potentials for carbon and nitrogen.

The experimental XA spectrum was measured at the dipole beamline PM3 at the synchrotron radiation source BESSY II in the Helmholtz-Zentrum Berlin. The sample solution was 1 $\mathrm{mol} / \mathrm{L} \mathrm{NiCl}_{2}(\mathrm{aq})$. The XA spectrum was measured from a thin liquid film in transmission mode using the experimental setup described in the literature. ${ }^{25}$

The simulated XA spectra of $\mathrm{Ni}^{2+}(\mathrm{aq})$ are presented in Figure 1 in comparison to our measured spectrum. In the calculation, RAS1 contains three $\mathrm{Ni} 2 \mathrm{p}$ orbitals and RAS2 contains five nominal $\mathrm{Ni} 3 \mathrm{~d}$ orbitals. No excitations to RAS3 were allowed. In the calculations we have explicit access to each electronic state, which is used in the assignment of the spectral features. First, the states are divided into $\mathrm{L}_{3}$ and $\mathrm{L}_{2}$ edges due to

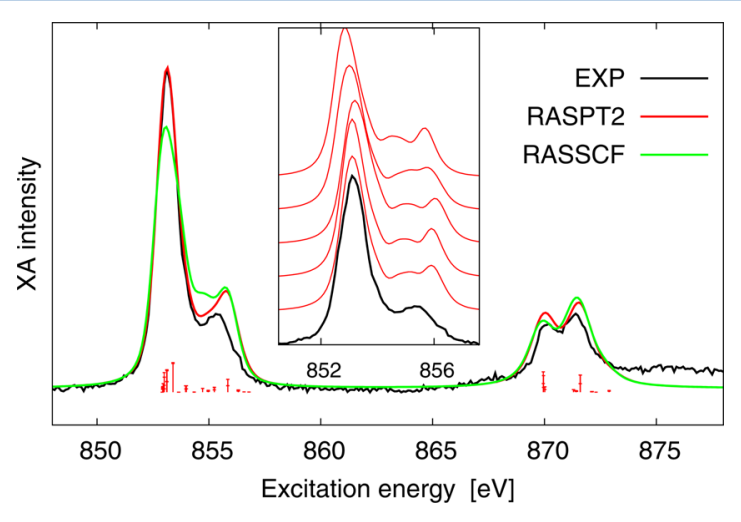

Figure 1. Comparison between the experimental XA spectrum of $1 \mathrm{M}$ $\mathrm{NiCl}_{2}(\mathrm{aq})$ solution and XA spectra calculated for $\mathrm{Ni}^{2+}\left(\mathrm{H}_{2} \mathrm{O}\right)_{6}$, with the RASSCF and RASPT2 methods. The calculations include core excitations arising from all $\left[(2 \mathrm{p})^{5},(3 \mathrm{~d})^{9}\right]$ permutations and the discrete RASPT2 transitions are shown. In the inset, spectra from a configurational sampling are presented. 
strong spin-orbit coupling of the $2 p$ core hole. Dominant features at each edge in the XA spectra correspond to different states belonging to the same electronic configuration $(2 \mathrm{p})^{5}(3 \mathrm{~d})^{9}$. Splittings and intensity ratios between the $\mathrm{L}_{3}$ and $\mathrm{L}_{2}$ edges are well reproduced. Second, both $\mathrm{L}_{3}$ and $\mathrm{L}_{2}$ are further split into two peaks. The low energy peak corresponds to triplet states with the $2 \mathrm{p}$ spin parallel to the unpaired $\mathrm{e}_{\mathrm{g}}$ electron spin. States in the higher energy peak have significant singlet character with antiparallel $2 \mathrm{p}$ and $\mathrm{e}_{\mathrm{g}}$ spins. The separation between triplet and singlet peaks is a result of 2p3d exchange interaction.

Exchange interaction also distorts the $\mathrm{L}_{3} / \mathrm{L}_{2}$ intensity ratio with respect to the expected $2: 1$ ratio in a system with pure $2 p$ spin-orbit interaction. At the high energy flank of the $\mathrm{L}_{3}$ triplet peak, states belonging to the $(2 \mathrm{p})^{5}\left(\mathrm{t}_{2 \mathrm{~g}}\right)^{5}\left(\mathrm{e}_{\mathrm{g}}\right)^{4}$ configuration show some intensity. These nominally double-excited states are shifted about one $10 \mathrm{Dq}$ value against the singly excited triplet peak, as expected $(10 \mathrm{Dq}=0.9 \mathrm{eV})$. The XA spectrum of $\mathrm{Ni}^{2+}(\mathrm{aq})$ is similar to that of $\mathrm{NiO}(\mathrm{s}),{ }^{12,26}$ although the latter shows a substructure in the features at $855-857 \mathrm{eV}$.

In the inset in Figure 1, the results from a sampling over configurations from an $\mathrm{MD}$ simulation are displayed. The spectra are obtained with a TZVP basis set, for which the splitting between the $\mathrm{L}_{3}$ and $\mathrm{L}_{2}$ edges are underestimated by 1.0 $\mathrm{eV}$ and within the edges there is also a small basis set dependence. In order to compensate for the additional limitations caused by the smaller basis set, the five uniformly distributed configurations, selected from the MD trajectory, were shifted by $-7.8 \mathrm{eV}$. Since we perform explicit configurational sampling, the Gaussian contribution to the broadening of these spectra contains only the part arising from experimental resolution. We observe fluctuations in the features of the selected spectra, showing that configurational sampling would be desirable. However, it is then important that the XA spectrum simulation gives a good description of the $2 p$ orbital energies relative to the valence orbitals, in order to yield accurate excitation energies.

Although the long-term goal is to combine this theoretical method with ab initio MD simulations for the study of photoinduced chemical reactions, an immediate motivation is provided by the suggestion of "dark-channel fluorescence" to explain fluorescence yield XA spectra for transition metals in solution, ${ }^{2}$ which was interpreted in terms of electron delocalization within the core-hole lifetime. ${ }^{2,27}$ In Figure 2 we display results of $\mathrm{Ni}^{2+}(\mathrm{aq})$ RIXS calculations.

The RIXS map in panel a in Figure 2 is based on the same RASPT2 calculations as the XA spectrum in Figure 1. Hence, the calculated RIXS final states correspond to excitations within the $\mathrm{Ni}(3 \mathrm{~d})^{8}$ configuration only (so-called ligand field excited states, LF). We label the LF states using spin-free labels in $O_{h}$ symmetry, although the calculation was done in $D_{2 h}$ symmetry with spin-orbit interaction included. The effect of the nonoctahedral symmetry of the complex and the spin-orbit interaction between $3 \mathrm{~d}$ electrons is weak: $\sim 0.1 \mathrm{eV}$. The valence states are thus built on the basis of determinants from three possible configurations: $\left(t_{2 g}\right)^{6}\left(e_{g}\right)^{2},\left(t_{2 g}\right)^{5}\left(e_{g}\right)^{3}$, and $\left(t_{2 g}\right)^{4}\left(e_{g}\right)^{4}$. Peaks at zero energy loss correspond to resonant elastic scattering with the final state being the ${ }^{3} \mathrm{~A}_{2 \mathrm{~g}}$ ground state of the complex. At the first XA resonance at the $\mathrm{L}_{3}$ edge (and also $\mathrm{L}_{2}$ edge), the most intense RIXS peak at $0.9 \mathrm{eV}$ energy loss is the ${ }^{3} \mathrm{~T}_{2 \mathrm{~g}}$ state. Note that the energy splitting between ${ }^{3} \mathrm{~A}_{2 \mathrm{~g}}$ and ${ }^{3} \mathrm{~T}_{2 \mathrm{~g}}$ is equal to $10 \mathrm{Dq}$. The experimental $10 \mathrm{Dq}$ value is $1.1 \mathrm{eV}$ in the $\mathrm{Ni}^{2+}(\mathrm{aq})$ complex; ${ }^{29}$ therefore we estimate the accuracy of

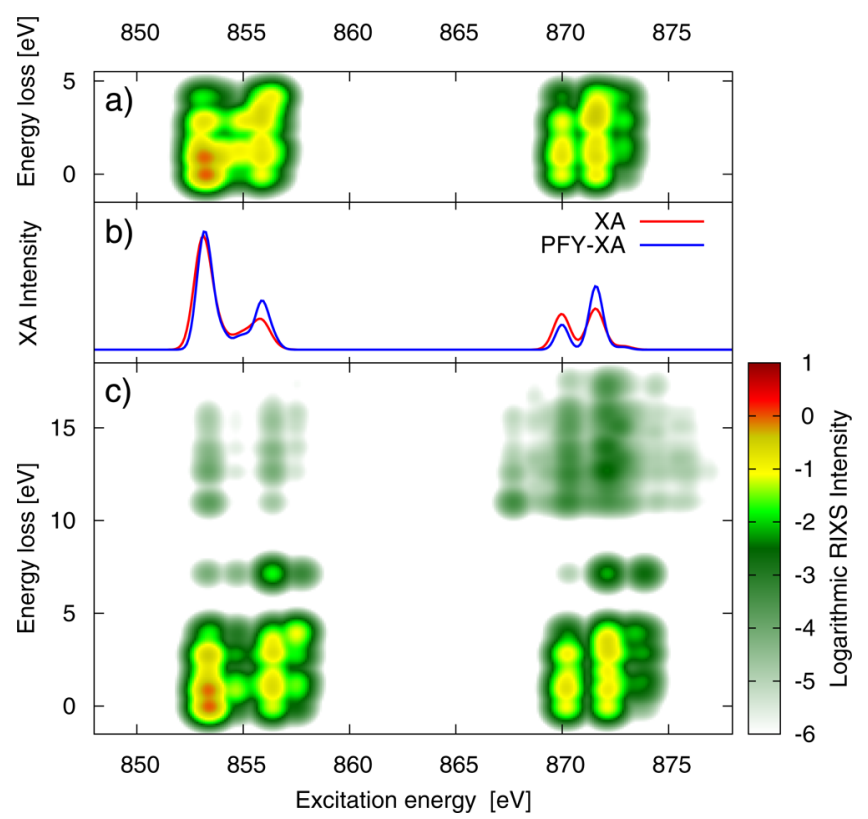

Figure 2. (a) The RIXS spectrum corresponding to the RASPT2 XA spectrum in Figure 1 was calculated according to the KramersHeisenberg expression, ${ }^{28}$ neglecting the polarization dependence, interference, and vibrational effects. (b) The PFY-XA spectrum was derived by integrating the RIXS spectrum over all calculated fluorescence decay channels for each excitation energy. (c) By extending the active space to ligand orbitals with two occupied $\mathrm{Le}_{\mathrm{g}}$ orbitals in RAS1 (at most one hole) and three $\mathrm{Lt}_{2 \mathrm{~g}}$ orbitals in RAS3 (at most one electron), we could also model charge-transfer (CT) excitations in the RIXS spectrum. (A logarithmic intensity scale is used in the RIXS maps to enhance weak peaks).

calculated valence state energies to be $0.2 \mathrm{eV}$. The second RIXS feature at the first $\mathrm{XA}$ resonance at $2.9 \mathrm{eV}$ energy loss corresponds to ${ }^{3} \mathrm{~T}_{1 \mathrm{~g}}$ states. The $\mathrm{XA}$ resonance at the highenergy side of the $L_{3}$ edge (and $L_{2}$ edge) has significantly more singlet character in relation to the first XA resonance, which has almost pure triplet character. This implies that the second XA resonance decays with higher intensity to singlet valence states. The RIXS feature around $\sim 2 \mathrm{eV}$ corresponds to ${ }^{3} \mathrm{~T}_{1 \mathrm{~g}},{ }^{1} \mathrm{E}_{\mathrm{g}}$ and ${ }^{1} \mathrm{~T}_{2 \mathrm{~g}}$ states, whereas the peak at $3.5 \mathrm{eV}$ is mostly the ${ }^{\mathrm{P}} \mathrm{T}_{1 \mathrm{~g}}$ state.

Each core excitation has a particular set of decay channels to valence-excited states, over which we can integrate to derive a fluorescence yield XA spectrum. The RASPT2 calculation in panel $\mathrm{b}$ in Figure 2 captures the qualitative differences between $\mathrm{XA}$ and partial fluorescence yield (PFY-XA). ${ }^{3,27}$ The relative enhancement in the PFY-XA spectrum of the "singlet" peaks is mainly due to a high number of available decay pathways, since both singlet and triplet valence-excited states can be reached. This is essential to consider when using L-edge fluorescence yield XA spectroscopy. However, for a detailed discussion on the interpretation of PFY-XA spectra, we refer to a recent study of the Cr L-edge X-ray spectra of $\mathrm{Cr}^{3+}(\mathrm{aq}),{ }^{30}$ in which the RASSCF method was successfully applied.

In panel $c$ in Figure 2, the result of the RIXS calculation with an extended active space is shown. The RAS1 active space now contains three $\mathrm{Ni} 2 \mathrm{p}$ orbitals and the two highest occupied nominal ligand orbitals of $\mathrm{e}_{\mathrm{g}}$ symmetry $\left(\mathrm{Le}_{\mathrm{g}}\right)$. RAS3 contains the three lowest unoccupied nominal ligand orbitals of $t_{2 g}$ symmetry $\left(\mathrm{Lt}_{2 \mathrm{~g}}\right)$. RAS2 includes the same orbitals as before (Ni $3 \mathrm{~d}$ ). Inclusion of nominal ligand orbitals $\mathrm{Le}_{\mathrm{g}}$ and $\mathrm{Lt}_{2 \mathrm{~g}}$ enables us to compute an extended range of excitations. In addition to LF 
states (configuration $\left.\left(\mathrm{Le}_{\mathrm{g}}\right)^{4}\left(\mathrm{t}_{2 \mathrm{~g}} \mathrm{e}_{\mathrm{g}}\right)^{8}\left(\mathrm{Lt}_{2 \mathrm{~g}}\right)^{0}\right)$, also states with the configurations $\left(\mathrm{Le}_{\mathrm{g}}\right)^{3}\left(\mathrm{t}_{2 \mathrm{~g}}, \mathrm{e}_{\mathrm{g}}\right)^{9}\left(\mathrm{Lt}_{2 \mathrm{~g}}\right)^{0}$ (ligand-to-metal charge transfer states, LMCT) and $\left(\mathrm{Le}_{\mathrm{g}}\right)^{4}\left(\mathrm{t}_{2 \mathrm{~g}}, \mathrm{e}_{\mathrm{g}}\right)^{7}\left(\mathrm{Lt}_{2 \mathrm{~g}}\right)^{1}$ (metal-toligand charge transfer states, MLCT) are calculated. Peaks above $10 \mathrm{eV}$ energy loss in panel $\mathrm{c}$ in Figure 2 correspond to LMCT and MLCT final states (the state at $\sim 7 \mathrm{eV}$ is a singlet ${ }^{1} \mathrm{~A}_{1 \mathrm{~g}}$ LF state, not presented in panel a). LMCT states can be best reached through the same XA resonances as LF states (excitation from Ni $2 p$ to $t_{2 g}$ or $e_{g}$ ) but MLCT states are most effectively reached through excitation from $\mathrm{Ni} 2 \mathrm{p}$ to $\mathrm{Lt}_{2 \mathrm{~g}}$. These XA states appear in the calculation starting from $867.5 \mathrm{eV}\left(\mathrm{L}_{3}\right.$ core-hole) and overlap with the lowest states in the $\mathrm{L}_{2}$ edge. The intensity of LMCT and MLCT peaks are considerably smaller than LF peaks due to small overlap of $\mathrm{Ni} 2 \mathrm{p}$ orbitals with $\mathrm{Le}_{\mathrm{g}}$ and $\mathrm{Lt}_{2 \mathrm{~g}}$ orbitals. In conclusion, the RIXS map in panel $c$ in Figure 2 contains both features from MLCT core excitations, indicated experimentally by the noisy XA feature in the interval 866-869 eV in Figure 1, and at high energy loss, weak features arising from $\mathrm{CT}$ valence-excitations related to decay channels opened by the solute-solvent interactions. ${ }^{2}$ By extending the active space, by introducing the $\mathrm{Le}_{\mathrm{g}}$ and $\mathrm{Lt}_{2 \mathrm{~g}}$ orbitals in RAS1 and RAS3, respectively, the orbitals are optimized in a different state averaging, which slightly perturbs the core-excitations by increasing the splitting within each Ledge.

For evaluation of the RIXS calculations in panel a in Figure 2, we display resonant $\mathrm{X}$-ray emission spectra in Figure 3. These

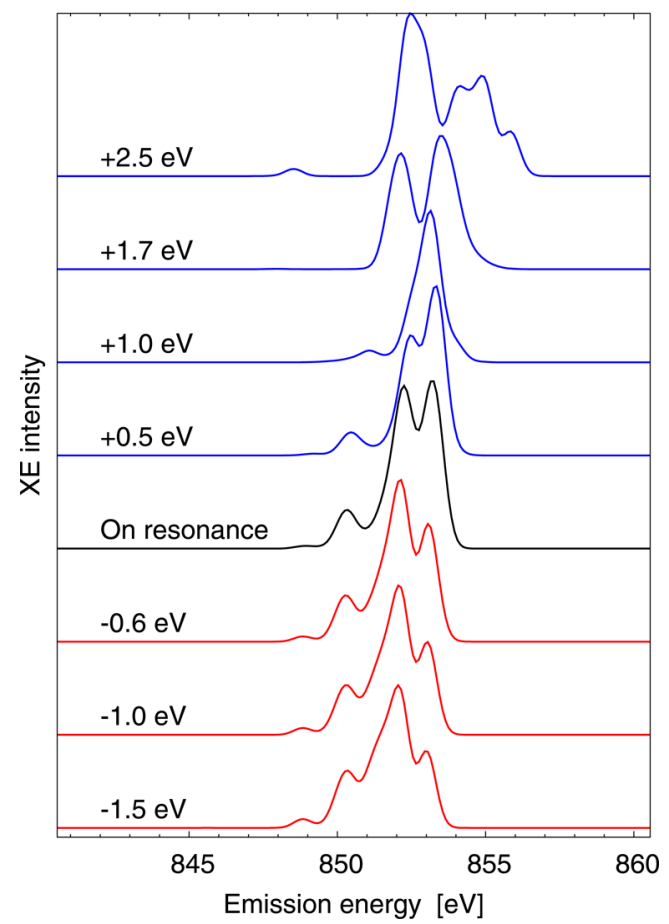

Figure 3. Resonant X-ray emission spectra of $\mathrm{Ni}^{2+}\left(\mathrm{H}_{2} \mathrm{O}\right)_{6}$ readily extracted from the RIXS map in panel a in Figure 2, for X-ray excitations energies detuned around the main $\mathrm{L}_{3}$ edge.

are vertical cuts in the RIXS map, and the energy scale is unambiguously determined by the calibration of the XA transitions. The detuning steps are chosen for an easy comparison to experimental data in Figure $3 \mathrm{~b}$ in a previous experimental study, ${ }^{3,27}$ taking into account the differences in calibration of the experimental XA spectra. The overall agreement is excellent, if we assume that there are remaining issues with the experimental calibration of the emission energy, which is the on-resonance excitation energy.

An attractive feature in the RASSCF calculations is that valence-excited states are treated on an equal footing with the electronic ground state. Thereby we are also able to study XA and RIXS spectra of systems in transient electronic states and highly distorted geometries. In this context, the theoretical development is a valuable complement to experimental investigations with time-resolved optical pump-X-ray probe techniques. $^{31}$ Time-resolved XA and RIXS with accurate theoretical analysis can be used to study changes in the local electronic structure with femtosecond resolution in reaction sites in biochemical processes or in situ in working photovoltaic devices. ${ }^{1}$ The applicability of the method to relevant investigations with time-resolved X-ray spectroscopy is proven by a direct comparison to experiments on polypyridyl iron complexes. $^{31}$ After photoexcitation of the low-spin singlet ground state of $\left[\mathrm{Fe}\left(\operatorname{tren}(\mathrm{py})_{3}\right)\right]^{2+}$, the complex evolve from a ${ }^{1}$ MLCT intermediate state into a long-lived high-spin quintet state. For interpretation of the transient X-ray absorption spectra of $\left[\mathrm{Fe}\left(\operatorname{tren}(\mathrm{py})_{3}\right)\right]^{2+}$, the methylated analogue $\mathrm{Fe}($ tren$\left.(6 \text {-methyl-py })_{3}\right)^{2+}$, which is high-spin at room-temperature, was experimentally employed. ${ }^{31}$ Computationally, we modeled the $\mathrm{XA}$ spectra of $\left[\mathrm{Fe}\left(\operatorname{tren}(\mathrm{py})_{3}\right)\right]^{2+}$ at different geometries and in different electronic states. X-ray absorption spectra from the singlet $\left({ }^{1} \mathrm{~A}_{1 \mathrm{~g}}\right)$ and quintet $\left({ }^{5} \mathrm{~T}_{2 \mathrm{~g}}\right)$ states were calculated with an active space consisting of the Fe $2 p$ orbitals with at most one hole in RAS1, and the nominal Fe 3d orbitals in RAS2. We added dynamic correlation to the singlet ground state and quintet valence-excited state to obtain a correct ordering of these states. The resulting correction, of a $0.77 \mathrm{eV}$ relative stabilization of the singlet state, was used in the XA spectrum calculations, presented in Figure 4.

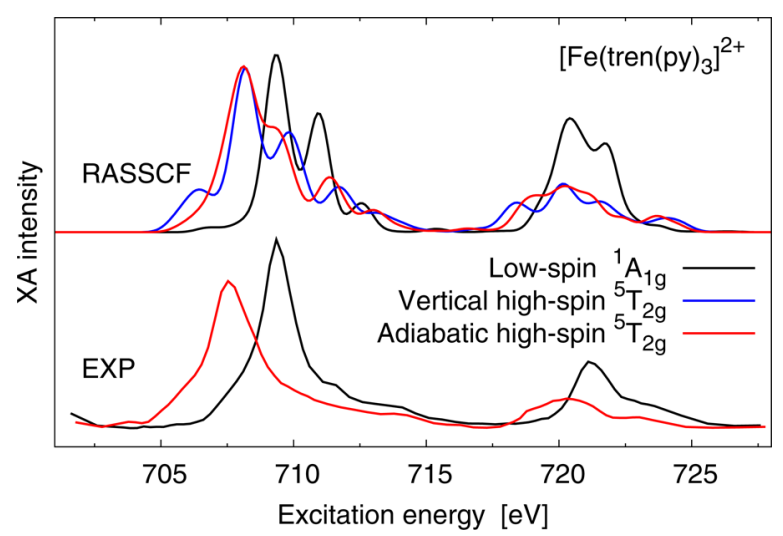

Figure 4. The calculated X-ray absorption spectra of $\left[\mathrm{Fe}\left(\operatorname{tren}(\mathrm{py})_{3}\right)\right]^{2+}$ is compared to the experimental XA spectra ${ }^{31}$ of $\left[\mathrm{Fe}\left(\operatorname{tren}(\mathrm{py})_{3}\right)\right]^{2+}$ (singlet ${ }^{1} \mathrm{~A}_{1 \mathrm{~g}}$ ) and a high-spin analogue (quintet ${ }^{5} \mathrm{~T}_{2 \mathrm{~g}}$ ). The RASSCF calculations include core excitations arising from all $\left[(2 \mathrm{p})^{5},(3 \mathrm{~d})^{7}\right]$ permutations. A uniform $0.9 \mathrm{eV}$ Gaussian broadening is employed for comparison to experiment.

Already with this minimal active space, the basic features are well described. The adequacy of the RASSCF method is apparent in the multiconfigurational character of the ground state singlet, the valence-excited quintet, and the core-excited states, which contain significant contributions from the determinants with $3 \mathrm{~d}_{2 \mathrm{~g}} \rightarrow \mathrm{e}_{\mathrm{g}}$ transitions. In the $\mathrm{e}_{\mathrm{g}}$ orbitals, the nominal Fe $3 \mathrm{~d}$ are strongly hybridized with the ligand $N \sigma$ 
lone-pairs. The shift of the main $\mathrm{L}_{3}$ edge, the appearance of prepeak related to the $3 \mathrm{~d} \mathrm{t}_{2 \mathrm{~g}}$ hole and the redistribution of intensity from the $\mathrm{L}_{2}$ edge can be reproduced. We also compare the XA spectra for a vertically and adiabatically valence-excited quintet state derived from geometries of the complex optimized in the low-spin and high-spin state. There is a smoothening of the spectral features as the $\left[\mathrm{Fe}\left(\operatorname{tren}(\mathrm{py})_{3}\right)\right]^{2+}$ complex relaxes in the quintet state due to an increase of the $\mathrm{Fe}-\mathrm{N}$ bonds from 2.018,2.045 $\AA$ to 2.20,2.30 A. A quantitative agreement would primarily require the inclusion of ligand orbitals in the active space, since there is a substantial covalent character in the metal-ligand bond. Together with ab initio MD simulations, this forms a framework for following the spectral evolution of photoinduced chemical reactions.

A careful study of the performance of the RASSCF/PT2 method will follow, in particular to other iron complexes, in which we will study MLCT and LMCT excitations and the covalent nature of the metal-ligand interaction. For the $\left[\mathrm{Fe}\left(\operatorname{tren}(\mathrm{py})_{3}\right)\right]^{2+}$, in particular, the inclusion of MLCT states will make it possible to determine the spectral signature in XA and RIXS of the ${ }^{1}$ MLCT intermediate directly following photoexcitation. $^{31}$

In conclusion, we have shown that ab initio multiconfigurational SCF calculations can give a highly reliable description of L-edge X-ray absorption (XA) and resonant inelastic X-ray scattering (RIXS) spectra of a transition metal in aqueous solution. This is of great value for the interpretation of experimental data as it allows for a unique discrimination of intra- versus interatomic effects. ${ }^{3,27}$ The nickel hexa-aqua complex is a very small and static model, and future investigations of transition metal ions in solution involve configurational sampling of larger cluster models, improvement of basis sets and extended active spaces. We also show that it can be applied both to more complex metallo-organic complexes. The scaling with system size is less severe than with respect to active space, and future investigations will determine its usefulness.

For L-edge X-ray spectroscopy of transition metal complexes, the method enables us to calculate (1) XA spectra from core excitations from $2 p$ core levels to $3 \mathrm{~d}$ valence levels, (2) RIXS spectra involving the subsequent decay into valence-excited states, (3) a proper description of XA and RIXS spectra also for highly distorted geometries and transient valence-excited states in time-resolved investigations.

\section{AUTHOR INFORMATION}

\section{Corresponding Author}

*E-mail: odelius@fysik.su.se. Phone: +46 (0)8 55378713. Fax: +46(0)855378601.

\section{Notes}

The authors declare no competing financial interest.

\section{ACKNOWLEDGMENTS}

I.J. and M.O. acknowledge Ulf Wahlgren for numerous discussions and crucial assistance, Roland Lindh for fruitful discussions, and Nils Huse for sharing experimental spectra. The work has financial support from the Swedish Research Council (VR), the Carl Trygger Foundation, and the Magnus Bergvall Foundation.

\section{REFERENCES}

(1) Van Kuiken, B. E.; Huse, N.; Cho, H.; Strader, M. L.; Lynch, M. S.; Schoenlein, R. W.; Khalil, M. Probing the Electronic Structure of a Photoexcited Solar Cell Dye with Transient X-ray Absorption Spectroscopy. J. Phys. Chem. Lett. 2012, 3, 1695-1700.

(2) Aziz, E. F.; Rittmann-Frank, M. H.; Lange, K. M.; Bonhommeau, S.; Chergui, M. Charge Transfer to Solvent Identified using Dark Channel Fluorescence-Yield L-Edge Spectroscopy. Nat. Chem. 2010, 2, 853-857.

(3) Aziz, E. F.; Eisebitt, S.; De Groot, F.; Chiou, J. W.; Dong, C.; Guo, J.; Eberhardt, W. Direct Contact Versus Solvent-shared Ion Pairs in $\mathrm{NiCl}_{2}$ Electrolytes Monitored by Multiplet Effects at $\mathrm{Ni}(\mathrm{II}) \mathrm{L}$ Edge X-ray Absorption. J. Phys. Chem. B 2007, 111, 4440-4445.

(4) de Groot, F.; Kotani, A. Core Level Spectroscopy of Solids (Advances in Condensed Matter Science); CRC Press Taylor \& Francis Group: Boca Raton, FL, 2008.

(5) Stavitski, E.; de Groot, F. M. The CTM4XAS Program for EELS and XAS Spectral Shape Analysis of Transition Metal L Edges. Micron 2010, 41, 687-694.

(6) Shirley, E. L. Bethe-Salpeter Treatment of X-ray Absorption including Core-Hole Multiplet Effects. J. Electron Spectrosc. Relat. Phenom. 2005, 144-147, 1187-1190.

(7) Vinson, J.; Rehr, J. J.; Kas, J. J.; Shirley, E. L. Bethe-Salpeter Equation Calculations of Core Excitation Spectra. Phys. Rev. B 2011, $83,115106 / 1-115106 / 7$.

(8) Laskowski, R.; Blaha, P. Understanding the $\mathrm{L}_{2 ; 3} \mathrm{X}$-ray Absorption Spectra of Early $3 d$ Transition Elements. Phys. Rev. B 2010, 82, 205104/1-205104/6.

(9) Krüger, P. Multichannel Multiple Scattering Calculation of $\mathrm{L}_{2: 3^{-}}$ edge Spectra of $\mathrm{TiO}_{2}$ and $\mathrm{SrTiO}_{3}$ : Importance of Multiplet Coupling and Band Structure. Phys. Rev. B 2010, 81, 125121/1-125121/6.

(10) Fronzoni, G.; Stener, M.; Decleva, P.; Simone, M. d.; Coreno, M.; Franceschi, P.; Furlani, C.; Prince, K. C. X-ray Absorption Spectroscopy of $\mathrm{VOCl}_{3}, \mathrm{CrO}_{2} \mathrm{Cl}_{2}$, and $\mathrm{MnO}_{3} \mathrm{Cl}$ : An Experimental and Theoretical Study. J. Phys. Chem. A 2009, 113, 2914-2925.

(11) Ikeno, H.; Mizoguchi, T.; Tanaka, I. Ab initio Charge Transfer Multiplet Calculations on the $\mathrm{L}_{2 ; 3}$ XANES and ELNES of $3 \mathrm{~d}$ Transition Metal Oxides. Phys. Rev. B 2011, 83, 155107/1-155107/ 13.

(12) Haverkort, M. W.; Zwierzycki, M.; Andersen, O. K. Multiplet Ligand-Field Theory Using Wannier Orbitals. Phys. Rev. B 2012, 85, 165113/1-165113/20.

(13) Malmqvist, P.-Å.; Rendell, A.; Roos, B. O. The Restricted Active Space Self-Consistent-Field Method, Implemented with a Split Graph Unitary Group Approach. J. Phys. Chem. 1990, 94, 5477-5482.

(14) Finley, J.; Malmqvist, P.-Å.; Roos, B. O.; Serrano-Andrés, L. The Multi-State CASPT2 Method. Chem. Phys. Lett. 1998, 288, 299-306.

(15) Karlström, G.; Lindh, R.; Malmqvist, P.-Å.; Roos, B. O.; Ryde, U.; Veryazov, V.; Widmark, P.-O.; Cossi, M.; Schimmelpfennig, B.; Neogrady, P.; Seijo, L. MOLCAS: A Program Package for Computational Chemistry. Comput. Mater. Sci. 2003, 28, 222-239.

(16) Douglas, N.; Kroll, N. M. Quantum Electrodynamical Corrections to Fine-Structure of Helium. Ann. Phys. 1974, 82, 89-155.

(17) Hess, B. Relativistic Electronic-Structure Calculations Employing a 2-Component No-Pair Formalism with External-Field Projection Operators. Phys. Rev. A 1986, 33, 3742-3748.

(18) Roos, B. O.; Lindh, R.; Malmqvist, P.-Å.; Veryazov, V.; Widmark, P.-O. Main Group Atoms and Dimers Studied with a New Relativistic ANO Basis Set. J. Phys. Chem. A 2004, 108, 2851-2858.

(19) Roos, B. O.; Lindh, R.; Malmqvist, P.-Å.; Veryazov, V.; Widmark, P.-O. New Relativistic ANO Basis Sets for Transition Metal Atoms. J. Phys. Chem. A 2005, 109, 6575-6579.

(20) Malmqvist, P.-Å.; Roos, B. O.; Schimmelpfennig, B. The Restricted Active Space (RAS) State Interaction Approach with SpinOrbit Coupling. Chem. Phys. Lett. 2002, 357, 230-240.

(21) Coster, D.; Kronig, R. D. L. New Type of Auger Effect and its Influence on the X-ray Spectrum. Physica 1935, 2, 13-24. 
(22) Ohno, M.; van Riessen, G. A. Hole-Lifetime Width: A Comparison between Theory and Experiment. J. Electron Spectrosc. Relat. Phenom. 2003, 128, 1-31.

(23) Car, R.; Parrinello, M. Unified Approach for Molecular Dynamics and Density-Functional Theory. Phys. Rev. Lett. 1985, 55, 2471.

(24) 1997-2001; CPMD, Copyright IBM Corp 1990-2004, Copyright MPI für Festkörperforschung Stuttgart 1997-2001.

(25) Schreck, S.; Gavrila, G.; Weniger, C.; Wernet, P. A Sample Holder for Soft X-ray Absorption Spectroscopy of Liquids in Transmission Mode. Rev. Sci. Instrum. 2011, 82, 103101/1-103101/ 10.

(26) Ikeno, H.; Tanaka, I.; Koyama, Y.; Mizoguchi, T.; Ogasawara, K. First-Principles Multielectron Calculations of $\mathrm{Ni}_{2 ; 3}$ NEXAFS and ELNES for $\mathrm{LiNiO}_{2}$ and Related Compounds. Phys. Rev. B 2005, 72, $075123 / 1-075123 / 8$

(27) Lange, K. M.; Könnecke, R; Ghadimi, S.; Golnak, R.; Soldatov, M. A.; Hodeck, K. F.; Soldatov, A.; Aziz, E. F. High Resolution X-ray Emission Spectroscopy ofWater and Aqueous Ions using the Micro-jet Technique. Chem. Phys. 2010, 377, 1-5.

(28) Kramers, H. A.; Heisenberg, W. Über die Streuung von Strahlung durch Atome. Z. Phys. A 1925, 31, 681-708.

(29) Landry-Hum, J.; Bussière, G.; Daniel, C.; Reber, C. Triplet Electronic States in $d^{2}$ and $d^{8}$ Complexes Probed by Absorption Spectroscopy: A CASSCF/CASPT2 Analysis of $\left[V\left(\mathrm{H}_{2} \mathrm{O}\right)_{6}\right]^{3+}$ and $\left[\mathrm{Ni}\left(\mathrm{H}_{2} \mathrm{O}\right)_{6}\right]^{2+}$. Inorg. Chem. 2001, 40, 2595-2601.

(30) Wernet, P.; Kunnus, K.; Schreck, S.; Quevedo, W.; Kurian, R.; Techert, S.; de Groot, F.; Odelius, M.; Föhlisch, A. Dissecting Local Atomic and Intermolecular Interactions of Transition-metal Ions in Solution with Selective X-ray Spectroscopy. J. Phys. Chem. Lett. 2012, 3, 3448-3453.

(31) Cho, H.; Strader, M. L.; Hong, K.; Jamula, L.; Gullikson, E. M.; Kim, T. K.; de Groot, F. M. F.; McCusker, J. K.; Schoenlein, R. W.; Huse, N. Ligand-field Symmetry Effects in Fe(II) Polypyridyl Compounds Probed by Transient X-ray Absorption Spectroscopy. Faraday Discuss. 2012, 157, 463-474. 